\title{
Sound therapy in children
}

\author{
Sukhbir K Shahid ${ }^{1}$ \\ ${ }^{1}$ Consultant Pediatrician and Neonatologist, Mumbai, India
}

*Corresponding Author: Dr. Sukhbir K. Shahid, Consultant Pediatrician and Neonatologist, Shahid Clinic, Ghatkopar (East), Mumbai-400 077. MH. India. Phone: 0091-9869036606,Email: s_kaur_shahid@yahoo.com

\section{Abstract}

Sound remains largely under-evaluated in the field of therapeutic medicine. It produces the desired changes both by the sound as well as the vibrational energy. Various modes have been used to deliver the energy to the individual. But studies on use of sound therapy in adults and children are limited and of poor design. In children, it has been tested in various conditions such as autism, developmental delays, in neonatal intensive care units on preterm babies, during MRI to lessen the anxiety and use of GA and sedatives, in ADHD, in pediatric cardiac care units, in childhood asthma, in emergency room patients, in chronic pain, neurological disabilities, and in troubled adolescents with substance abuse or neurotic disorders. The outcomes have been generally positive. But more large-scale and properly-designed comparative studies would be required before the low cost technology and safe sound therapy could be incorporated in the therapeutic armamentarium in children.

Keywords: Children, Pediatric care, Sound therapy.

\section{INTRODUCTION}

Sound is a vibroacoustic wave with diagnostic and therapeutic potential. But this has not been tapped enough and sound therapy in medicine remains largely underexplored. There are very few studies with small sample sizes done in this field and most of these are in adults [1, 2]. Nonetheless, results seem promising. Hence more extensive and large-scale studies are required for this unique modality to find a place in mainstream therapeutics.

Some studies exist that conclude that sonic therapy is beneficial for children with neurological, cognitive, psychosomatic, developmental, musculoskeletal, and such disorders ${ }^{[3]}$. The current review discusses this aspect of sound as a complementary therapy in children with health issues.

\section{REFRESHING THE PHYSICS OF SOUND}

Sound, the sensation experienced through the special sense and organ of hearing, is a vibration that is transmitted as an acoustic wave through a solid, liquid, or gaseous medium. Acoustic waves with frequencies lying between $20 \mathrm{~Hz}$ to $20 \mathrm{KHz}$ are audible to human ear (audio frequency). Above and below this range limit are the ultrasound and infrasound waves respectively ${ }^{[4]}$. Thus sound is an oscillation and is propagated as a wave motion in an elastic media or air. It vibrates the media and the latter shows pressure, particle displacement, and velocity variations. But the media per se does not travel with the sound wave. The sound waves travel faster in solids than liquids or air. They excite the hearing mechanism (organ of Corti) and this is perceived as sound by the human ear. In simple description, sound is a sinusoidal plane wave with a frequency (waves per unit time), wavelength (inverse of frequency), amplitude, speed, and direction. Sound waves get reflected, refracted, or even attenuated by the transmitting media. This is dependent on the density, pressure, motion, and viscosity of the medium ${ }^{[5]}$.

\section{SOUND THERAPY: MECHANISM OF ACTION IN DISEASES}

Sound has frequency and amplitude and travels in a particular direction with a particular speed in that medium. It transmits its waves into a substance and makes it vibrate to its frequency. The substance particles have their own distinctive frequencies. On contact with the sound, they begin to vibrate to the tune of the sound waves.

Since ancient times, the sound therapists believe that during a disease, the body energy frequencies go out of tune. For 
instance, the normal body has a frequency of $50-100 \mathrm{~Hz}$. But in an autistic child, this frequency might go as high as $200-500 \mathrm{~Hz}$ leading to hyperactivity and other symptoms. But, pure sound produced by various gadgets can align these deranged frequencies and bring them back to normal. This is said to help in mitigation of the problem and bring relief to the diseased individual ${ }^{[6-9]}$.

Various neuro-imaging techniques have shown how relaxing or stimulating sound and music produce specific brain wave changes on EEG ${ }^{[9,10]}$. At the higher level, therapy in the form of sound can induce a sedative phase wherein theta waves predominate in the brain. This assists healing, creative and clear thinking, and enhances memory, awareness, and intuition [11, 12]. It is hypothesized that neural regenerative processes are enhanced ${ }^{[13]}$. It relaxes and removes stress and pain. Effect is deeper and prolonged [14].

The sound affects changes not only via the hearing mechanism and action on the brain areas but also by its vibratory role on the body parts. Placement of the sound-producing tools on the body regions passes these vibrations into the body tissues for the desired action. This leads to synchronization and equalization of the bodily frequencies and harmony ${ }^{[3,15]}$.

It has physical, mental, emotional, and claimed spiritual actions on the body and is thus supposedly a wholesome therapy. It can form a complementary therapy with other modes of treatment and is generally safe ${ }^{[16]}$.

The actions of sound are on the nervous system (brain electrochemistry) and also locally on various organs (body physiology). Thus it is a mind-body intervention. The main physiological actions include:

1. Endorphin release and action on nervous tissue for a relaxing effect. Negative emotions are lessened and positivity and well-being increases [17].

2. Improvement of circulation and blood flow to body parts [18].

3. Insomnia, headaches, migraines are reduced $[19,20]$.

4. Joint and muscle and menstrual pains come down ${ }^{[14]}$.

5. Digestive problems are decreased [21-23].

6. Self-healing and immunity is boosted [24].

7. Good sound liberates the emotional trauma and problems $[6,25]$.

8. Right and left brain synchronization is improved [26].

9. Cognition, memory, thinking is improved [27].

10. Energy level and well-being are improved [28, 29].

This all is also called 'psychoneuroimmunology'; a term that the American psychologist Robert Ader coined in the year 1981 [30]. It is used to imply the interactions that take place between psychology, neurology, and immunology in a person. These interplay is a complex one and also bilateral. This could be utilized to positively influence one's body through positive and good thinking.
SOUND THERAPY VS SOUND HEALING AND THE TOOLS

Sound therapy is also termed audio therapy. When sound is organized into music, it is called music therapy. Besides, there are also recorded relaxation talks and Nature sounds (such as flowing water, leaves rustling) that could be utilized for the purpose of sound therapy. Various tools are used for the purpose to produce melodious and harmonious sounds and include Himalayan or Tibetan singing bowls, cymbals, crystal singing bowls, gongs, drums, bells, tuning forks, and binaural beat therapy. The binaural beat therapy is still semiexperimental and used for stress relief and treatment of anxiety [31]. These sounds are usually pre-recorded and available on CDs or any instrument that can play the sounds. They can be also performed live. This form of therapy is the 'receptive' or 'passive' sound therapy and can be administered self by the individual or may be facilitated by a practitioner. The other form is the 'active' or 'expressive' sound therapy in which the person self-produces the sound vocally or by means of a musical instrument ${ }^{[32]}$. Self-reading of poetry or fiction with the intent to experience a therapeutic action is called bibliotherapy and is also a form of sound therapy [33]. When vibrating tools are applied directly to the body, it is vibroacoustic therapy. Low frequency sinusoidal waves in the $30-120 \mathrm{~Hz}$ range assist in reducing rigidity and tremors in Parkinsonism and increase the speed of walking ${ }^{[34]}$. Similar benefits are observed of this lowfrequency sound stimulation (LFSS) in fibromyalgia, Alzheimer's disease, and depression [7]. The circulation and movement is improved and pain and rigidity is lessened. The vibrations are administered as beds, mattresses, pillows, mats, chairs, wristbands, wearable backpacks and the like. In them, the sound files are played by means of transducers, bass shakers, or exciters. This has been tried in flat foot in children with good outcomes [3]. Thus sound therapy can be made prescribable and dosable in clinics and other centers.

Sound healing (Sonic healing) is similar but the term is reserved when the benefits are claimed to be more spiritual and explained on that basis. This includes Leonard Horowitz's Solfeggio frequencies and sound bath sessions. Creative visualization, guided imagery are components of such therapy [35]. But everything said and done, harmonious sound does have benefits on the physiology, psychology, and social life of a person.

\section{USES AND STUDIES IN CHILDREN}

In adults, specific or organized sound form has been tried in several medical conditions with variable outcomes. These include cognitive and emotional problems, diminished conscious levels, neurological conditions, physical deformities, pain, cancer, fertility issues, to boost immunity, and for COPD. It has also been found to be useful in grief and bereavement ${ }^{[36]}$. 
A number of studies of sound therapy have been carried out in preterm babies in NICU [37]. Researchers noticed an improvement in physiological outcomes such as oxygen saturation, heart rate, respiratory rate, and blood pressure as well as in behavioural state (body movements, facial expression, crying) and in the pain scores. Evidence also reveals that music has positive impact in the long-term such as on duration of hospitalization, non-nutritive sucking, and weight gain. Lullabies have been found to soothe preterm babies and affect positively their sleep and eating [38, 39]. Scientists have also tried with sounds of womb fluids (Remo Ocean Disk) and two-tone heart beat rhythms (Gato box) on premature babies 32 weeks and older with positive outcomes. Their heart rates normalized and alert state quietness increased. They found that sucking was more with heartbeat rhythm and womb sounds enhanced sleep. For sound therapy administration, various techniques were employed. Live or recorded music with or without kangaroo care was found to improve oxygenation, regulate respiration, and decrease neonatal distress. It was found that female voice had better outcome [40]. Pacifier-activated lullaby device was also used and decreased heart rate, respiratory rate, and improved sleep patterns and sucking behavior. Multimodal stimulation (MMS) using auditory, tactile, vestibular, and visual stimulation led to earlier discharge from the neonatal intensive care unit. Music can also mask the disruptive noises of the NICU and lead to infant stimulation and enhance parent-child bonding. Music has also been tried in cardiac ICUs for infants and it led to a decrease heart and respiratory rate and blood pressure ${ }^{[41]}$.

In case of toddlers or older children, they either listen to the music or sound or play with the instrument that emits the sound such as the singing bowls. The positive benefits are observed no matter what mode is used to reach the sound to the child. This applies particularly to autistic children who had appreciable positive impact with this therapy. It was seen that the hyperactivity and stereotypical behaviour lessened and their attention and focus improved [42]. The obedience increased and there was an improvement noticed in their verbal and non-verbal communication. Similar benefits were reported for other children with special needs ${ }^{[43-45]}$. Sound has also been found to help ADHD children. These children have raised catecholamines and music helped them to have better concentration and lesser hyperactivity White noise (a random signal that has equal intensity at different frequencies) was useful and probably helped via stochastic resonance [46]. Music therapy also has benefits in pediatric emergency room patients who had less distress and less pain during intravenous insertion when music played in the background. The health care providers also felt that it was easier to do IV insertion in the music group patients ${ }^{[47]}$.

During MRI in children, sedatives and GA are used to calm the anxious and crying child. Though effective, these suffer from their own side-effects. To minimize their use, sound therapy is one of the various non-pharmacological techniques tried to pacify the child. MRI-compatible audiovisual systems have been found to be useful. The other methods employed include better MRIs to decrease both scan times and motion artifacts, noisereduction methods, optimization of the MRI environment, involvement of child life specialists, feed-sleep manipulation of the child, feed-and-bundle and distraction techniques, play therapy, photo diaries, mock scanners, use of infant incubators/immobilizers, sucrose solutions, guided imagery, and artificial intelligence ${ }^{[48,49]}$. Sound studies in older children with physical trauma have revealed that it lessens pain and hastens the rehabilitation in them ${ }^{[50]}$. Research in chronic pain in children revealed that they had lesser stress and music helped draw their attention away from it. Post-neurological damage rehabilitation process was accelerated with sound therapy and the lung function and speech clarity of such children improved drastically. It has also been found to offer some help in children with psychosomatic disorders such as bronchial asthma [unpublished observations of Dr. Shahid SK]. Vibroacoustic therapy has immense benefits in flat foot therapy ${ }^{[3]}$.

French ENT surgeon Alfred Tomatis was working with sound for hearing impaired people and realized that the same sound led to visible changes in the mood and well-being of the person. The research was expanded in light of these new findings and now specific frequencies are delivered therapeutically to the brain via air and bone conduction depending on the person's disease, its severity, and duration of illness [51]. This is now being used for children for autistic spectrum disorders, behaviour issues, Down syndrome babies, learning disabilities, motor control problems, reading and writing delays, and speech delays with variable results ${ }^{[52]}$.

Music therapy has been tried with good outcomes in troubled adolescents [53-56]. In children and adolescents with major depressive and anxiety disorders, the severity of the internalizing symptoms came down ${ }^{[57]}$.

Sound therapy is also found to be a safe supplementary remedy in children. The research avenues for it are endless and need to be properly assessed. It can complement conventional therapy in cardiovascular ailments and also in major mental disorders but cannot substitute it. It is advisable to be careful in using this therapy in children with epileptic seizures lest the sound precipitates the seizure attack or worsen restlessness in them. Also, there is a possibility that it might aggravate headaches in some migraine patients.

\section{CONCLUSION}

Sound therapy in its various forms; alone or in combination and in pure natural forms or artificially produced does show some promise in the treatment of various diseases in children. Its role in these conditions should be further evaluated and it could find a place as a major complementary mode in pediatric therapeutics. Its usefulness is immense but proper studies directed in the direction of revealing its hidden potential could reap benefits for child health.

Conflicts of Interest: The author declares that no source of support was used for this article and there is no association with any product or subject.

Acknowledgements: None 


\section{REFERENCES}

1. Lopez-Gonzalez MA, Lopez-Fernandez R. Sequential sound therapy in tinnitus. Int Tinnitus Journal 2004; 10(2):150-155

2. Ugras GA, Yildirim G, Yuksel S, Ozturkcu Y, Kuzdere M, Oztekin SD. The effect of different types of music on patients' preoperative anxiety: A randomized controlled trial. Complement Ther Clin Pract 2018; 31:158-163

3. Iodice P, Bellomo RG, Migliorini M, Megna M, Saggini R. Flexible flatfoot treatment in children with mechanical sound vibration therapy. Int J Immunopathol Pharmacol. 2012; 25(1 Suppl):9S-15S

4. Berg RE, Stork DG. The Physics of Sound, $2^{\text {nd }}$ Edition. New Jersey, USA: Prentice Hall; 1995

5. Vitale A, Giampaolo F (Editors). Sound waves: Propagation, Frequencies and Effects (Acoustics Research and Technology). New York, USA: Nova Science Pub Inc.; 2011

6. Lyendo TO. Exploring the effect of sound and music on health in hospital settings: A narrative review. Int J Nurs Stud. 2016; 63:82 100.

7. Lili N, Heidi A, Pasqualino M, Lee B. The effect of low-frequency sound stimulation on patients with fibromyalgia: A clinical study. Pain Res Manag 2015; 20 (1):e21-e27.

8. Ward LM. Synchronous neural oscillations and cognitive processes. Trends Cogn Sci. 2003; 7:553-559.

9. Ahn IS, Kim BY, You KB, Bae MJ. A study on the characteristics of an EEG Based on a Singing Bowl's Frequency. In Lee R (Ed) Software Engineering Research, Management and Applications. SERA Studies in Computational Intelligence. Volume 789. New York, USA: Springer, Cham.; 2018. https://doi.org/10.1007/978-3319-98881-8_16

10. Ramirez R, Planas J, Escude N, Mercade J, Farriols C. EEG-based analysis of the emotional effect of music therapy on palliative care cancer patients. Front Psychol. 2018; 9: article 254, 7 pages.

11. Lee E-J, Bhattacharya J, Sohn C, Verres R. Monochord sounds and progressive muscle relaxation reduce anxiety and improve relaxation during chemotherapy: a pilot EEG study. Complement Ther Med. 2012; 20(6):409-416

12. Kučikienè $D$, Praninskienè $R$. The impact of music on the bioelectrical oscillations of the brain. Acta Med Litu. 2018; 25(2):101-106. doi:10.6001/actamedica.v25i2.3763

13. Fukui $\mathrm{H}$, Toyoshima $\mathrm{K}$. Music facilitate the neurogenesis, regeneration and repair of neurons. Medical Hypotheses. 2008; 71:765-769

14. Lee JH. The Effects of Music on Pain: A Meta-Analysis. J Music Ther. 2016; 53(4):430-477. doi: 10.1093/jmt/thw012.

15. Reybrouck M, Podlipniak P, Welch D. Music and Noise: Same or Different? What Our Body Tells Us. Front Psychol. 2019; 10: article1153, 13 pages. doi:10.3389/fpsyg.2019.01153

16. Witusik A, Pietras T. Music therapy as a complementary form of therapy for mental disorders. Pol Merkur Lekarski. 2019; 47(282):240-243

17. Qiu J, Jiang Y-F, Li F, Tong Q-H, Rong H, Cheng R. Effect of combined music and touch intervention on pain response and $\beta$ endorphin and cortisol concentrations in late preterm infants. BMC Pediatr. 2017; 17(1):38

18. Nakamura S, Sadato N, Oohashi T, Nishina E, Fuwamoto $Y$, Yonekura $Y$. Analysis of music-brain interaction with simultaneous measurement of regional cerebral blood flow and electroencephalogram beta rhythm in human subjects. Neurosci Lett. 1999; 275(3):222-226

19. Loewy J. Music Therapy as a potential intervention for sleep improvement. Nat Sci Sleep. 2020; 12:1-9

20. Risch M, Scherg H, Verres R. Musiktherapie bei chronischen Kopfschmerzen. Evaluation musiktherapeutischer Gruppen für Kopfschmerzpatienten [Music therapy for chronic headaches. Evaluation of music therapeutic groups for patients suffering from chronic headaches]. Schmerz. 2001; 15(2):116-125. German. doi: 10.1007/s004820170035. PMID: 11810342.

21. Camilleri M. Evolving concepts of the pathogenesis of irritable bowel syndrome: to treat the brain or the gut? J Pediatr Gastroenterol Nutr. 2009; 48 Suppl 2: S46-48. doi: 10.1097/MPG.0b013e3181a1174b

22. Shen YA. Complementary and alternative medicine for treatment of irritable bowel syndrome. Can Fam Physician. 2009; 55(2):143148

23. Lackner JM, Mesmer C, Morley S, Dowzer C, Hamilton S. Psychological treatments for irritable bowel syndrome: a systematic review and meta-analysis. J Consult Clin Psychol. 2004; 72(6): $1100-1113$

24. Núñez MJ, Mañá $P$, Liñares $D$, Riveiro MP, Balboa J, SuárezQuintanilla J, et al. Music, immunity and cancer. Life Sci. 2002; 71(9):1047-1057. doi: 10.1016/s0024-3205(02)01796-4

25. Pezzin LE, Larson ER, Lorber W, McGinley EL, Dillingham TR. Music-instruction intervention for treatment of post-traumatic stress disorder: a randomized pilot study. BMC Psychol. 2018; 6(1):60. doi: 10.1186/s40359-018-0274-8

26. Abrams DA, Ryali S, Chen T, Chordia P, Khouzam A, Levitin DJ, et al. Inter-subject synchronization of brain responses during natural music listening. Eur J Neurosci. 2013; 37(9):1458-1469. doi: 10.1111/ejn.12173

27. Ward EV, Isaac A, Donnelly M, Van Puyvelde M, Franco F. Memory improvement in aging as a function of exposure to mood-matching music. Acta Psychol (Amst). 2021; 212:103206. doi: 10.1016/j.actpsy.2020.103206

28. Creech A, Hallam S, Varvarigou M, McQueen H, Gaunt H. Active music making: a route to enhanced subjective well-being among older people. Perspect Public Health. 2013; 133(1):36-43. doi: 10.1177/1757913912466950

29. Wulff V, Hepp P, Wolf OT, Balan P, Hagenbeck C, Fehm T, et al. The effects of a music and singing intervention during pregnancy on maternal well-being and mother-infant bonding: a randomised, controlled study. Arch Gynecol Obstet. 2021; 303(1):69-83. doi: 10.1007/s00404-020-05727-8

30. Zachariae R. Psychoneuroimmunology: a bio-psycho-social approach to health and disease. Scand J Psychol. 2009; 50(6):645651. doi: 10.1111/j.1467-9450.2009.00779.x.

31. Beauchene C, Abaid N, Moran R, Diana RA, Leonessa A. The effect of binaural beats on verbal working memory and cortical connectivity. J Neural Eng. 2017; 14(2):026014. doi: 10.1088/17412552/aa5d67

32. Bruscia KE. Defining music therapy. Gilsum, New Hampshire, the USA: Barcelona Publishers; 1998.

33. Lehr F. Bibliotherapy. Journal of Reading. 1981; 25(1):76-79

34. King LK, Almeida QJ, Ahonen H. Short-term effects of vibration therapy on motor impairments in Parkinson's disease. Neurorehabilitation. 2009; 25(4):297-306.

35. Joseph Sonya. Sound Healing using Solfeggio frequencies. Graduate degree thesis at American College of Healthcare Sciences. June 2019. Downloaded on $6^{\text {th }}$ April 2021from https://www.researchgate.net/pub;ication/333852911_Sound_Hea ling_using_Solfeggio_Frequencies.

36. Ellen Zambo Anderson. Energy Therapy. In: Judith E. Deutsch and Ellen Zambo Anderson (Eds.). Complementary Therapies for Physical Therapy. USA; Elsevier Inc.; 2008. Pages 196-205.

37. Pölkki T, Korhonen A. The effectiveness of music on pain among preterm infants in the neonatal intensive care unit: a systematic review. JBI Libr Syst Rev. 2012; 10(58):4600-4609.

38. Novotney A. Music as Medicine. 2013; 44(10): Page 46. Downloaded on $6^{\text {th }} \quad$ April 2021 from https://www.apa.org/monitor/2013/11/music

39. Loewy J, Stewart K, Dassier AM, Telsey A, Homel P. The effects of music therapy on vital signs, feeding, and sleep in premature infants. Pediatrics. 2013; 131:902-918.

40. Schlez A, Litmanovitz I, Bauer S, Dolfin T, Regev R, Arnon S. 
Combining kangaroo care and live harp music therapy in the neonatal intensive care unit setting. The Israel Medical Association Journal 2011; 13(6):354-358

41. Yurkovich J. The Effect of music therapy entrainment on physiologic measures of infants in the cardiac intensive care unit: single case withdrawal pilot study Journal of music therapy 2018; 55(1):62-82

42. Bruscia KE, Grocke DE. Guided imagery and music: the Bonny method and beyond. Gilsum, New Hampshire, the USA: Barcelona Publishers; 2002.

43. Kim Y. The early beginnings of Nordoff-Robbins music therapy. J. Music Ther. 2004; 41(4):321-329.

44. Voigt M. Orff music therapy: an overview. Voices: A World forum for Music Therapy November 2003; 3(3). https://doi.org/10.15845/voices.v3i3.134

45. Janzen TB, Thaut MH. Rethinking the role of music in the neurodevelopment of autism spectrum disorder Music and Science 2018; $\quad 1$ : 2059204318769639. https://doi.org/10.1177/2059204318769639

46. Jackson NA. A survey of music therapy methods and their role in the treatment of early elementary school children with ADHD. J Music Ther. 2003; 40(4):302-323.

47. Hartling L, Newton AS, Liang Y, Jou H, Hewson K, Klassen TP, Curtis S. Music to reduce pain and distress in the pediatric emergency department: a randomized clinical trial. JAMA Pediatr. 2013; 167(9):826-835.

48. Dong SZ, Zhu M, Bulas D. Techniques for minimizing sedation in pediatric MRI J Magn Reson Imaging. 2019; 50(4):1047-1054.

49. McGuirt D. Alternatives to Sedation and General Anesthesia in Pediatric Magnetic Resonance Imaging: A Literature Review. Radiol Technol. 2016; 88(1):18-26.

50. Stanley P, Ramsey D. Music therapy in physical medicine and rehabilitation. Australian Occupational Therapy Journal 2012; 47(3):111-118

51. Thompson BM, Andrews SR. An historical commentary on the physiological effects of music: Tomatis, Mozart and neuropsychology. Integr Physiol Behav Sci. 2000; 35(3):174-188.

52. Corbett BA, Shickman K, Ferrer E. Brief report: the effects of Tomatis sound therapy on language in children with autism. $J$ Autism Dev Disord. 2008; 38(3):562-566.

53. Swedberg Yinger, Olivia, Gooding, Lori. Music therapy and music medicine for children and adolescents. Child and Adolescent Psychiatric Clinics 2014; 23(3):535-553

54. Keen MSocSc, Alexander W. Using music as a therapy tool to motivate troubled adolescents. Social Work in Health Care 2005; 39:3-4(3-4):361-373

55. Porter S, McConnell T, McLaughlin K, Lynn F, Cardwell C, Braiden $\mathrm{HJ}$, et al. Music therapy for children and adolescents with behavioural and emotional problems: a randomized controlled trial. J Child Psychol. Psychiatry 2017; 58(5):586-594.

56. Bednarz LF, Nikkel B. The role of music therapy in the treatment of young adults diagnosed with mental illness and substance abuse. Music Therapy Perspectives 1992; 10:21-26

57. Geipel J, Koenig J, Hillecke TK, Resch F, Kaess M. Music-based interventions to reduce internalizing symptoms in children and adolescents: a meta-analysis. Journal of Affective Disorders 2018; 225:647-656. 